\title{
An Analysis on the Diurnal Variation of the Terrestrial Magnetism, especially on the day-time-variation of geomagnetically quiet days*
}

\author{
By Masaziro OTA \\ (Aso Magnetic Observatory, Kyoto University)
}

\begin{abstract}
The average feature of the geomagnetic diurnal-variation is conventionally described by the Fourier coefficients. Camparing these coeffs. of quite days and with those of all days, the characteristics of the $S_{q}$-variation are discussed. It is recognized that at the middle and the low latitudes the amount of variation on quite days is large during the day-time and negligible during the night. If we plot a scheme which varies during the day-time and not during the night, then the average feature of the $S_{q}$-variation bears a striking resemblance to this scheme. The feature of this scheme is derived from the Fourier coeffs. computed by the conventional method, and their latitudinal distributions are clarified by using a peculiar latidude. Moreover, this scheme is very satisfactory with the potential.
\end{abstract}

\section{Introduction}

Recent studies on the geomagnetic variation in connection with electrical properties of the upper atmosphere have realed that the geomagnetic variation observed at the earth's surface is a synthetic effect of various properties of the upper atmosphere and the earth's interior. In closely examining the results of world-wide observations, local effects of the observatories and unexpected anomalies of the $S_{q}$-field are recognized. Then, if we want to discuss more delicate features and properties of the upper atmosphere, we must re-examine the geomagnetic data which have been used. For example: As Dr. Rikitake [1] has pointed out regarding the anomalous behaviour of the geomagnetic variations of short period, there are to be found some peculiar regions. And, Prof. Hasegawa [2] has utilized distribution-maps of the Fourier coeffs. of the geomagnetic $S_{q}$-variation as a method for its analysis. According to these maps, we can see several stations where observational data do not match that of the neibouring stations. Moreover, the East-component ( $\mathrm{Y}$ ) at Huancayo shows the same phase as the North-component (X) and a comparatively large value relative to the Magnetic Equator. This fact suggests the requirement of selecting other axes for the geomagnetic diurnal-variation.

* This paper is a contribution of Geophysical Paper dedicated to Prof. M. Hasegawa on his sixtieth birth-day, January 2, 1954. 
The writer has examined the data of the geomagnetic diurnal-variation of the middle and the low latitudes during the II Polar Year, using the diagrams of the latitudinal distribution of the Fourier coeffs. about the condition of the annual mean $1 / 2$ (Summer plus Winter). In the present analysis, the geomagnetic variation-fields are not developped by the spherical function, but are shown by the latitudinal distributions of the Fourier coeffs. applyed for the local time (L'T); because, we must calculate the terms of higher degree to converge the series.

The present paper is aimed at the re-examination of the feature of quiet days, because the above mentioned facts may include the effect of a disturbing field in spite of the feature of quiet days. First of all, the apparent distinction between fhe diurnal variation of quiet days and that of disturbed days is introduced by comparing with the respective Fourier coefficients of each observatory. In conclusion, the writer defines the feature of the geomagnetic $S_{q}$-variation by a simple method.

2. Apparent distinction between the geomagnetic diurnal-variation of quiet days and disturbed days

Fig. 1 shows the observatories referred to this paper which are distributed within the latitudes of $60^{\circ} \mathrm{N}$ and $60^{\circ} \mathrm{S}$. By the table of the Fourier coeffs. of diurnal variation of those stations (not shown in this paper), it can be seen that the first,

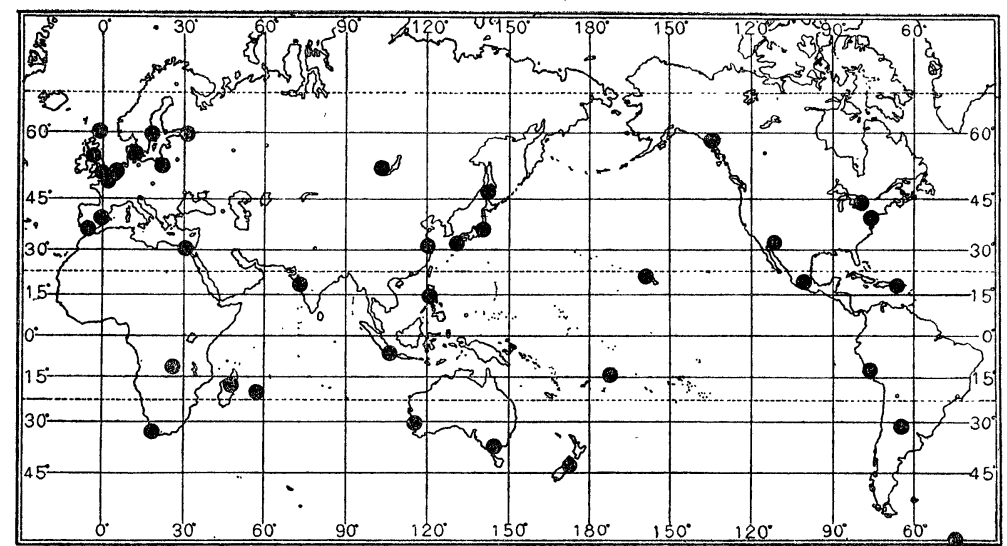

Fig. 1 The Observatories distributed within $60^{\circ} \mathrm{N}-60^{\circ} \mathrm{S}$. (Geographical coordinates)

the second and the third harmonics are predominant and that the terms higher than the fourth are negligible. Therefore, the feature of the diurnal variation can be represented by these terms, which are discussed in this paper. Figs. 2-5 show the latitudinal distribution of each term of the North-(X) and East-(Y) components in regard to quiet days and all days (and disturbed days partly). The feature of diurnal variation of all days seems to show the average state of disturbed days. The mean of diurnal variation of the International Disturbed Days is sometimes affected by a largely disturbed day or successive disturbance owing to a peculiar magnetic-storm, and then the feature of the diurnal variation is highly distorted. Therefore, the average feature of the diurnal variation of disturbed days is to be 

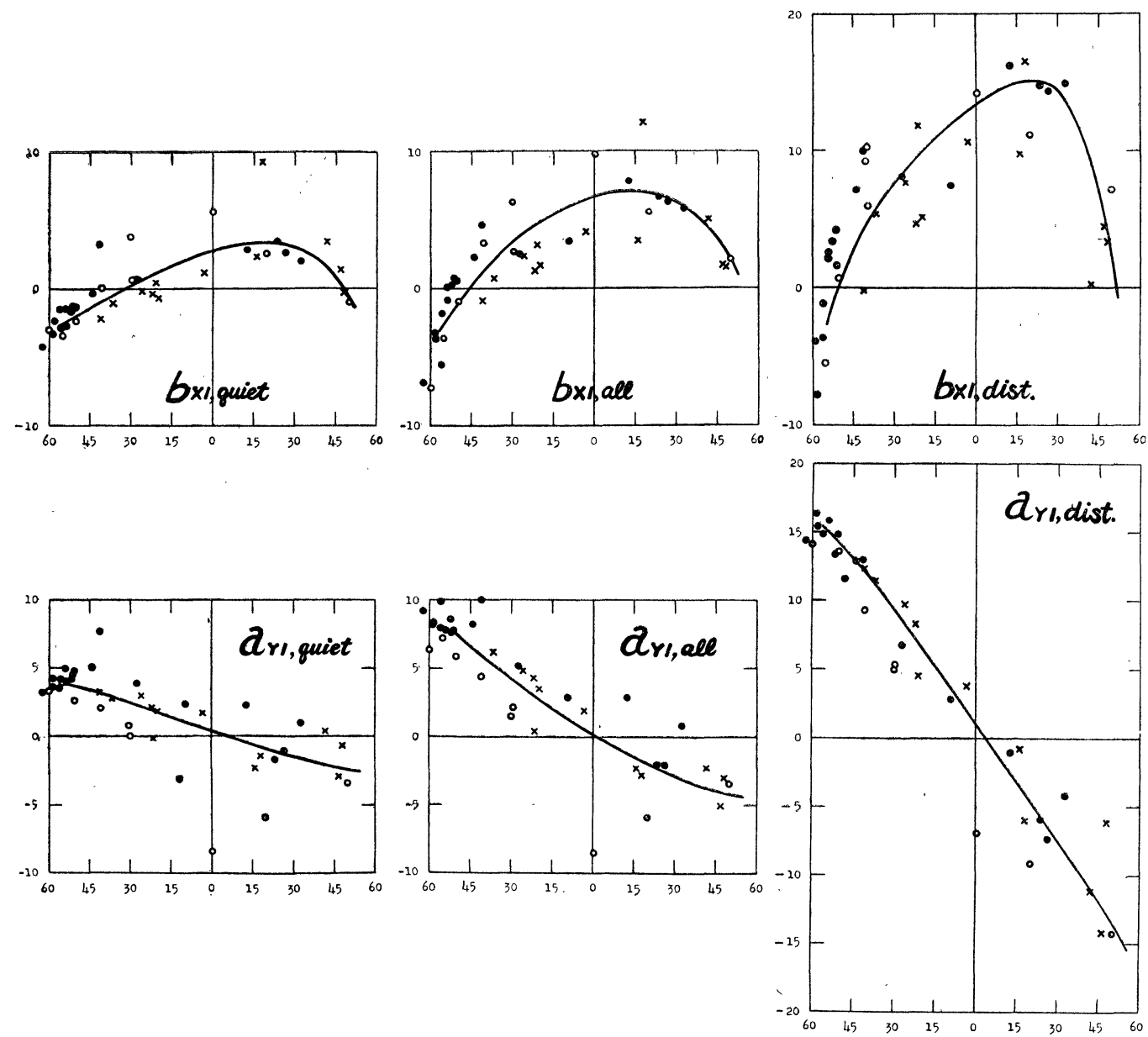

Fig. 2 Latitudinal distributions of $b_{X 1}$ and $a_{Y 1}$.

dealt with the all days' mean, but that of quiet days will be defined and represented by the International Quiet Days. In the diagrams of Figs. 2-7, the abscissa denotes the geomagnetic latitude and the ordinate denotes the force whose unit is $10^{-5} \mathrm{c} . \mathrm{g} . \mathrm{s}$. in geographical components. The discrepancy between the geographical components and the geomagnetic ones of magnetic force is insignificant so far as the data of the middle and the low latitudes are concerned.

The question whether the geographical, geomagnetic or other latitudes are to be selected in reference to the locations of the observatories is discussed in the following paragraph. The object of this selection is to show the same value at the same latitude of different longitudes. According to Hasegawa's analysis [2] the geomagnetic latitude is better than the geographical one for this end. In Figs. 2-5, in regard to the latitudinal distributions of the coeffs., it will be noticed that the definite distinction between quiet days and disturbed days appears in one term in respect to one component, i.e., the sine-term of the first harmonic of X-comp. $\left(b_{X 1}\right)$ and the cosine-term of the first harmonic of Y-comp. $\left(a_{Y 1}\right)$. About the other terms 

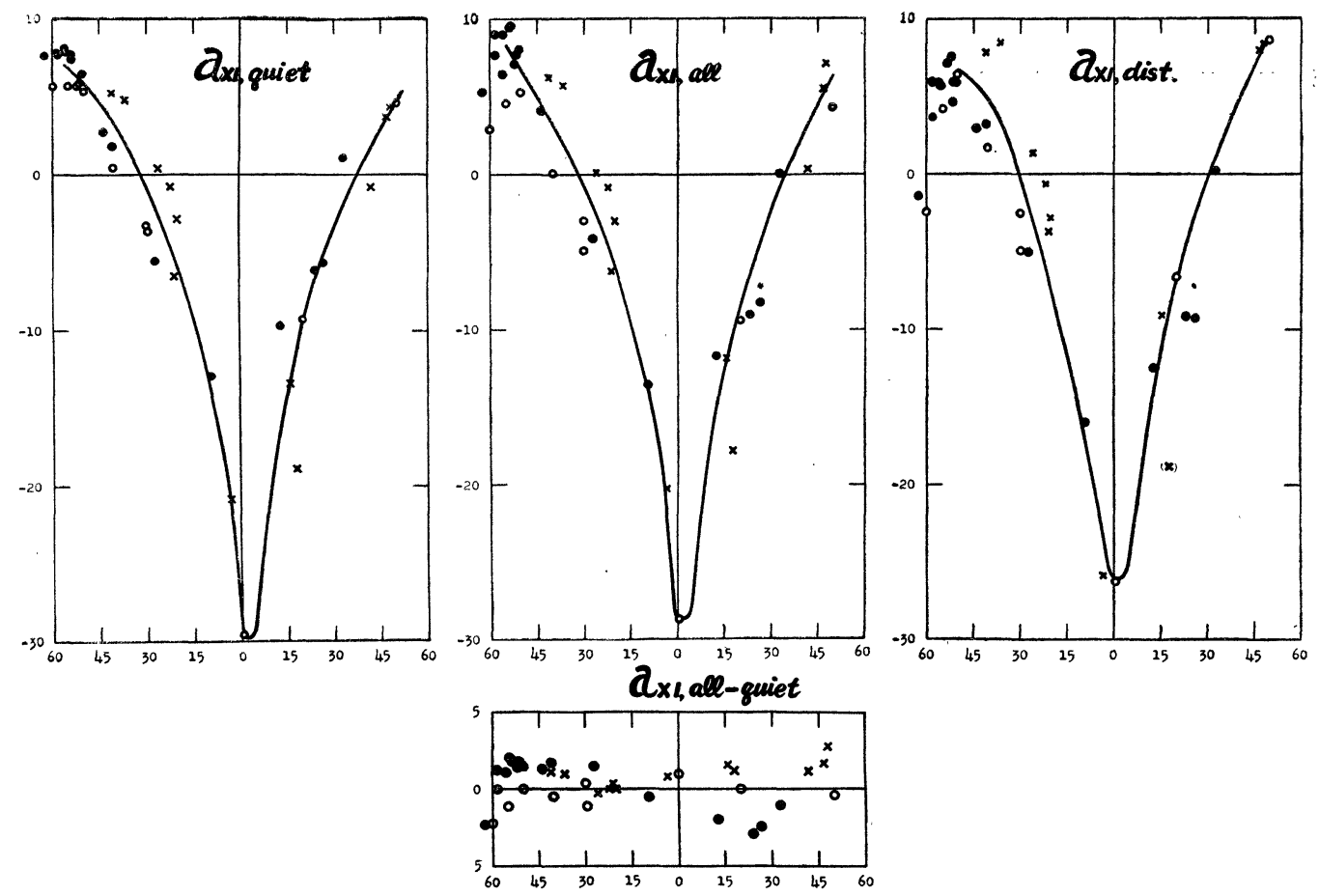

Fig. 3a Latitudinal distributions of $a_{X 1}$.
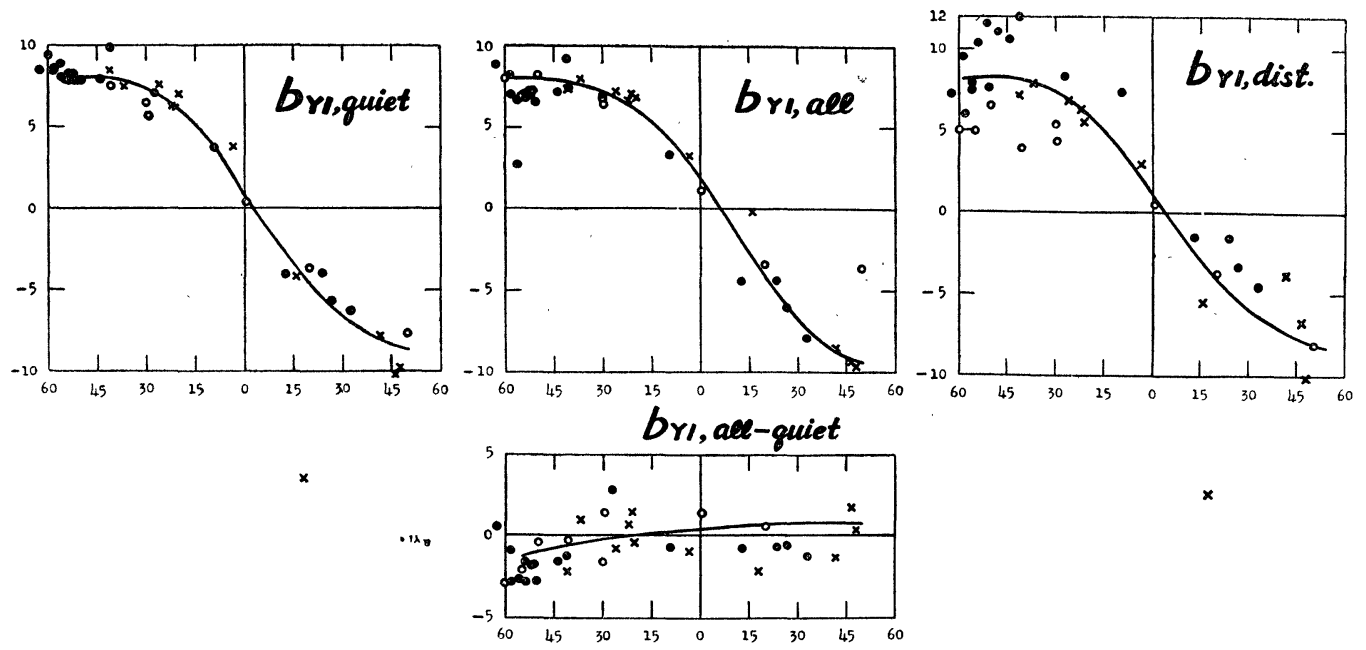

Fig. 3b Latitudinal distributions of $b_{Y 1}$. 
we cannot detect any distinctions; as shown in Figs. 3 and 4, difference-values (all minus quiet) are negligible compared with their original values, or, the latitudinal distributions of the difference-values are rather similar to those of the quiet, though they should be similar to those of the disturbed. The diagram of $b_{X 1}$ and $a_{Y 1}$ of the disturbed days is more distinct than that of all days as shown in Fig. 2. By this treatment, $b_{X 1}$ and $a_{Y 1}$ can be said to be the terms of the disturbing field. Then we may adopt this term as a measure of the disturbance (SD). But this term takes a certain value even on quiet days and shows a similar diagram to that of the disturbed days as we see in Fig. 2. On the other hand, this term of quiet days should have a significance of the quiet state. About this contradiction, further treatment will follow in the next paragraph.

\section{The feature of the $S_{q}$-variation centred at $11^{\mathrm{h}}$ in local time}

The diurnal-variation-field of the quiet days is represented by the equivalent current-vortex. According to Ota's paper [3], the centre of this vortex moves around the earth with the sun and is situated at the middle latitude along the meridian of of about $11^{\mathrm{h}}$ in local time (LT). This fact means that the mode of the variation at the middle latitude is symmetric about $11^{\mathrm{h}}$ in LT. And, as mentioned in the previous paragraph, the sine-term of the first harmonic of the $\mathrm{X}$-comp. takes a finite
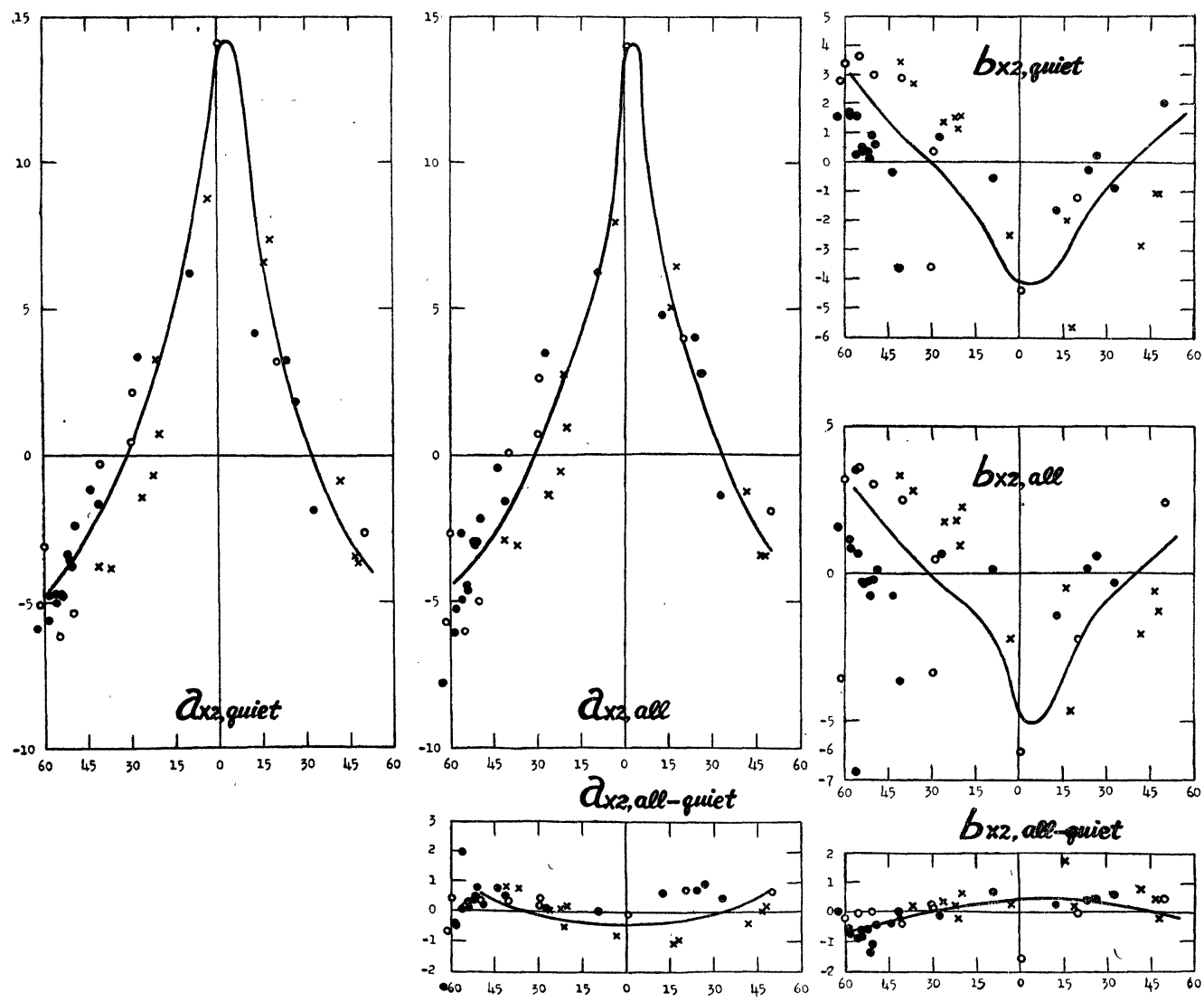

Fig. $4 \mathrm{x}$ Latitudinal distributions of $a_{X 2}$ and $b_{X 2}$. 

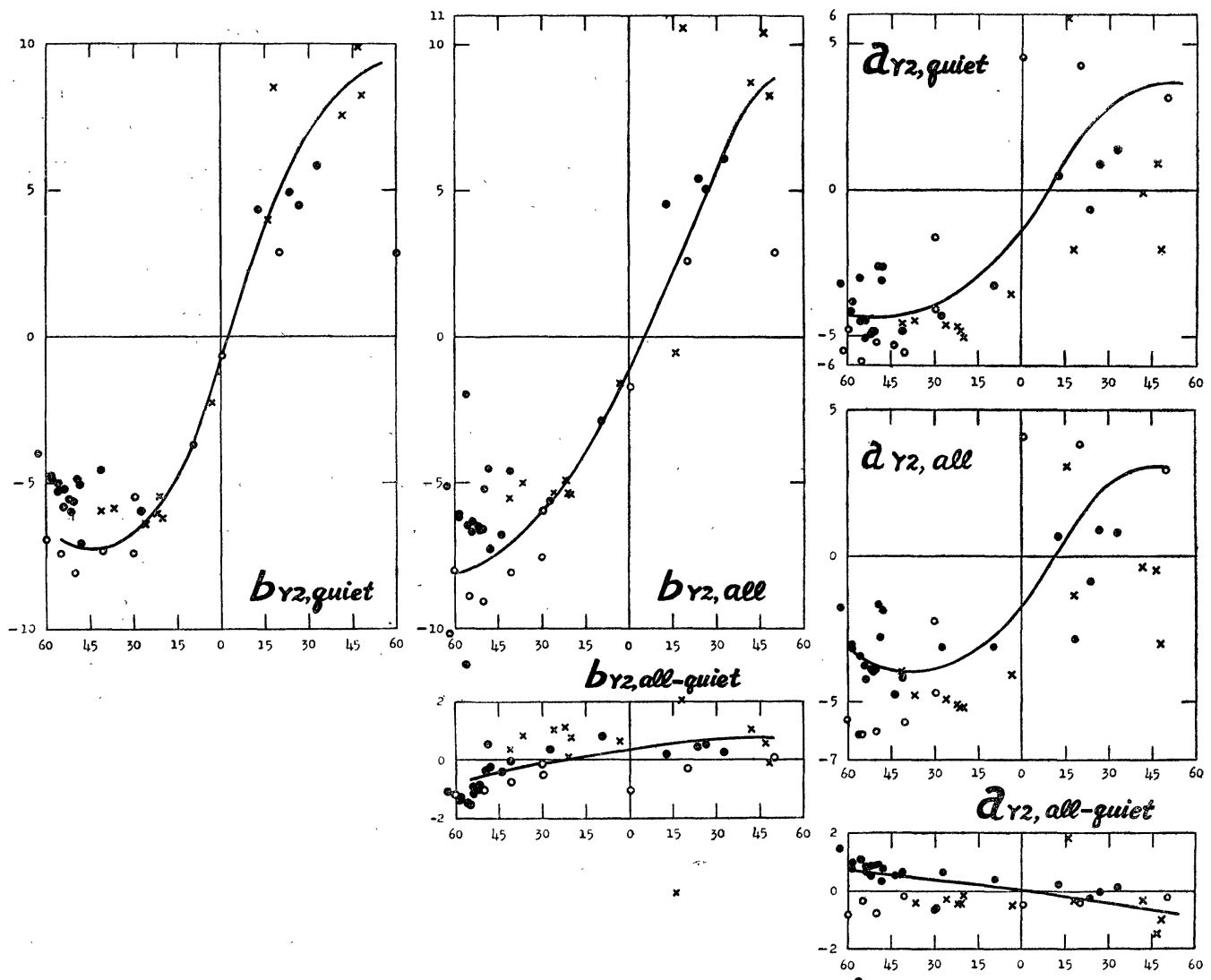

Fig. $4 \mathrm{y}$ Latitudinal distribution of $a_{Y 2}$ and $b_{Y 2}$.
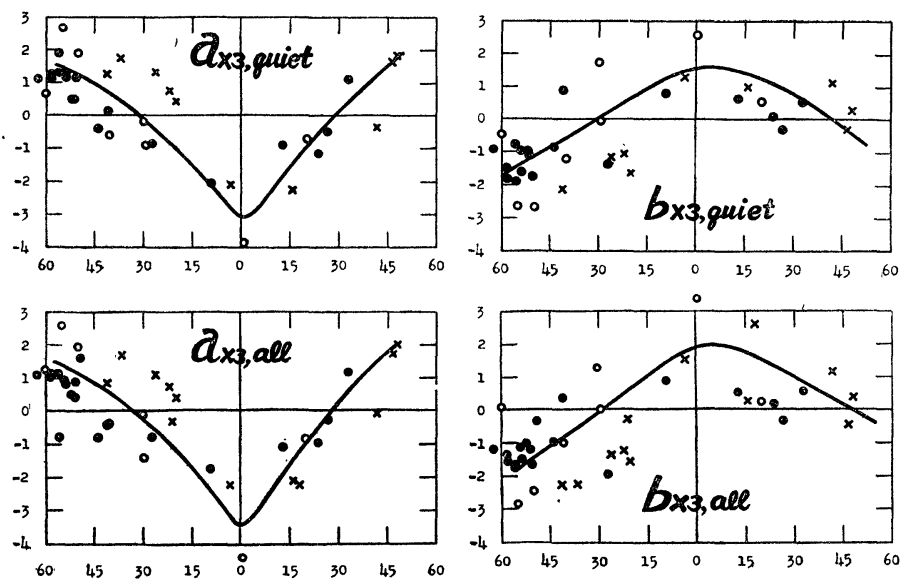

Fig. 5 Latitudinal distributions of $a_{X 3}$ and $b_{X 3}$. 
value on the quiet day. If we consider a variation centred at $11^{\mathrm{h}}$ in $\mathrm{LT}$. instead of at noon, this (asymmetric) term would almost vanish, though some value would remain due to error. (The situation of this centre is not necessarily on the meridian of $11^{\mathrm{h}}$ in LT.)

As already discussed, $b_{X 1}$ can be taken as a measure of SD, the fact that $b_{X 1}$ of the quiet days has a finite value is due to a phase-lag of the variation which is centred at noon. Figs. 6 and 7 is the latitudinal distribution of Fourier coeffs. computed through such a treatment and can be expressed as follows:

where

$$
a_{m} \cos m t+b_{m} \sin m t=a_{m}^{\prime} \cos m t^{\prime}+b^{\prime}{ }_{m} \sin m t^{\prime}
$$

$$
\begin{aligned}
& t^{\prime}=t+15^{\circ}=t+1^{\mathrm{h}} \\
& m=1,2 \text { and } 3
\end{aligned}
$$

As seen in Figs. 6 and 7, the sine-terms of all harmonics of the X-comp. $\left(b^{\prime}{ }_{X 1}, b^{\prime} X^{2}\right.$ and $\left.b_{X 3}^{\prime}\right)$ and the cosine-terms of all harmonics of the Y-comp. $\left(a_{Y 1}^{\prime}, a_{Y 2}^{\prime}\right.$ and $\left.a_{Y 3}^{\prime}\right)$
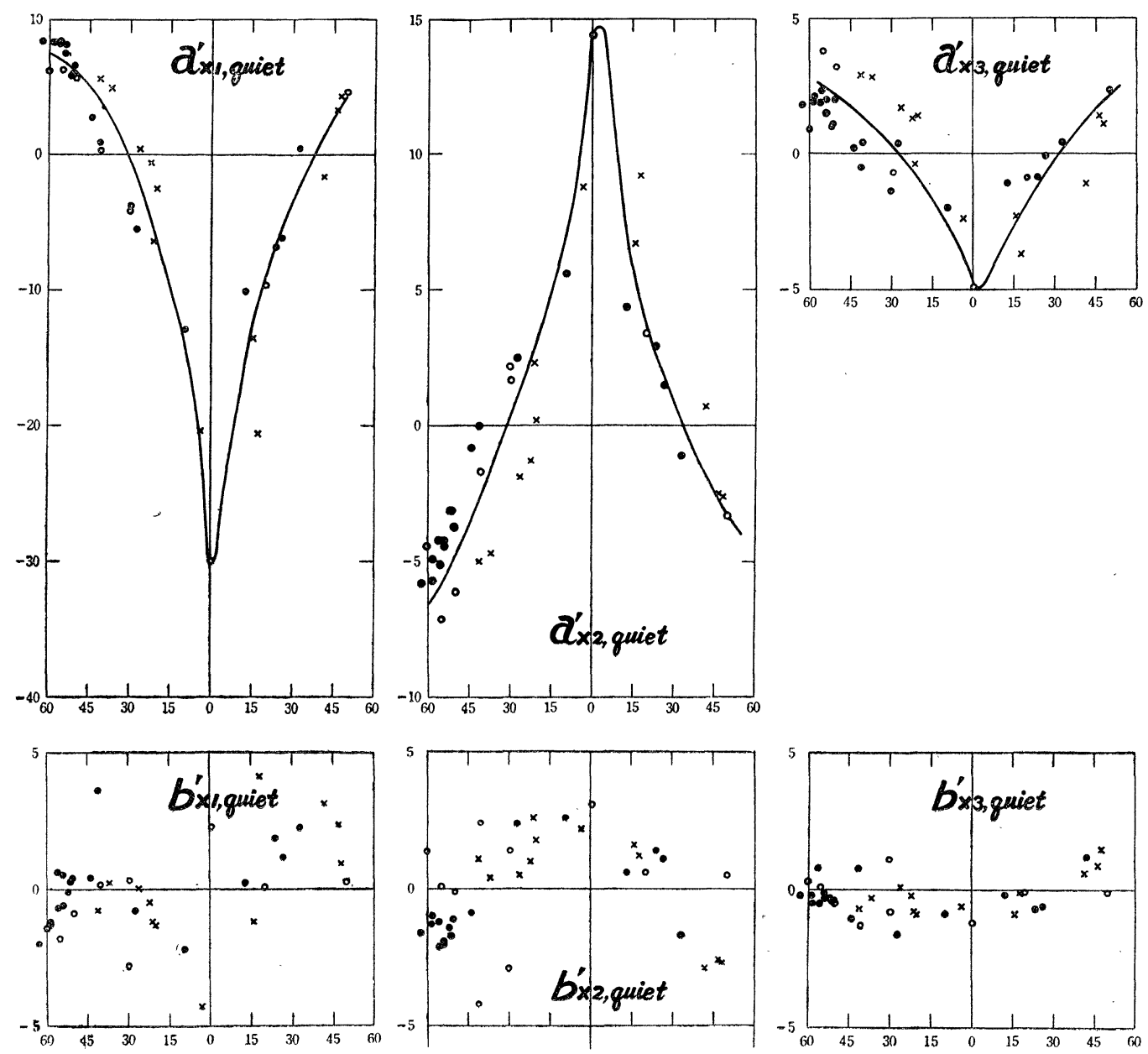

Fig. 6 Latitudinal distributions of $a^{\prime} x^{\prime}$ s and $b^{\prime} x^{\prime}$ s. (Fourier coeffs. of X-component centred at $11^{\mathrm{h}}$ LT.) 

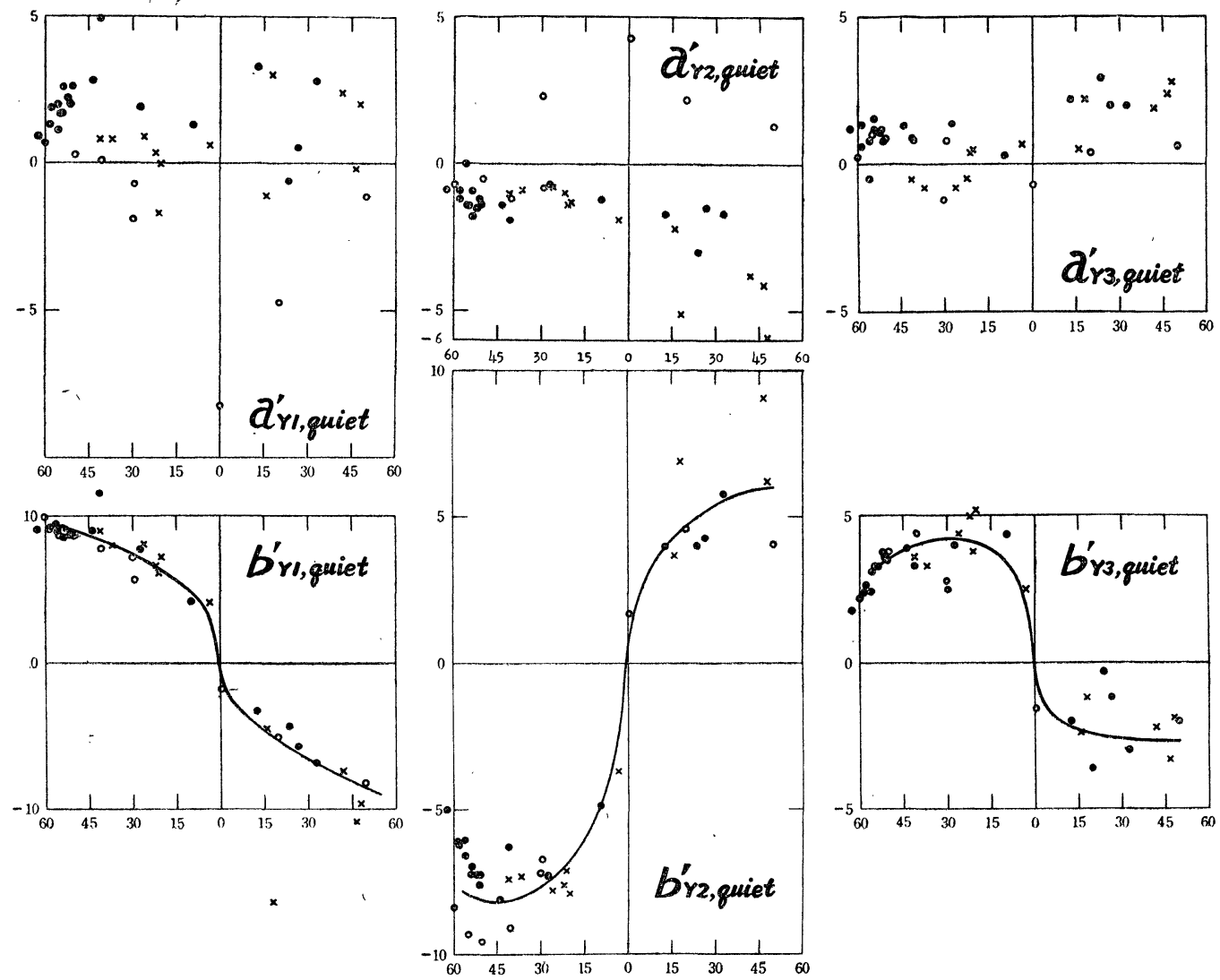

Fig. 7 Latitudinal distributions of $a_{Y}^{\prime}{ }_{Y}^{\prime} s$ and $b^{\prime}{ }_{Y}^{\prime}$ 's (Fourier coeffs. of Y-component centred at $11^{\mathrm{h}} \mathrm{L}$.. T)

do not show any systematic feature concerning the latitudinal distribution. For the scattering of points on the diagrams, the writer wishes to conclude that they are caused by regional anomalies of the $S_{q}$-field. In conclusion of this paragraph, the $S_{q}$-variation is shown by $a_{X}^{\prime}$ 's and $b_{Y}^{\prime}$ 's.

\section{Selection of the equator}

In the present paper, the writer does not consider the longitudinal inequalities of the $S_{q}$-variation, and deals with the latitudinal distribution of coeffs. only. It is assumed that the features of the diagrams of the distributions are quite similar for all longitudes, and the geomagnetic coordinates are favourable for this assumption as the first approximation. But, precisely speaking about the latitudinal distribution, some discrepancies are seen in the different zones; in Figs. 2-8, the black circles denote the stations in the European Zone, the white circles those in the American Zone and the crosses those in the Pacific Zone. Consequently, we wish to find another coordinate which is more agreeable for the different zones. Judging from the discrepancies between the different zone, this investigation will be resolv. ed by the selection of the equatorial point where the amount of variation reaches a decisive maximum. By Hirono's theory [4] the range of the diurnal variation 
takes maximum at a point where magnetic dip is equal to zero, and shows a sharp gradient at about $5^{\circ} \mathrm{N} \& \mathrm{~S}$ distance from this point, as to the same meridian. Then, as a test-case of this investigation, the Magnetic Equator (where dip is zero) is adopted, i.e., the cross-point of the Magnetic Equator and the geomagnetic meridian is taken as $0^{\circ}$ latitude and measured by degree along the geomagnetic meridian. This method is also adopted by $H$. Maeda [5]. But this measure of coordinates does not satisfy the function of spherical surface, but is nearly satisfactory in the middle and the low latitudes. Fig. 8 are diagrams obtained by such
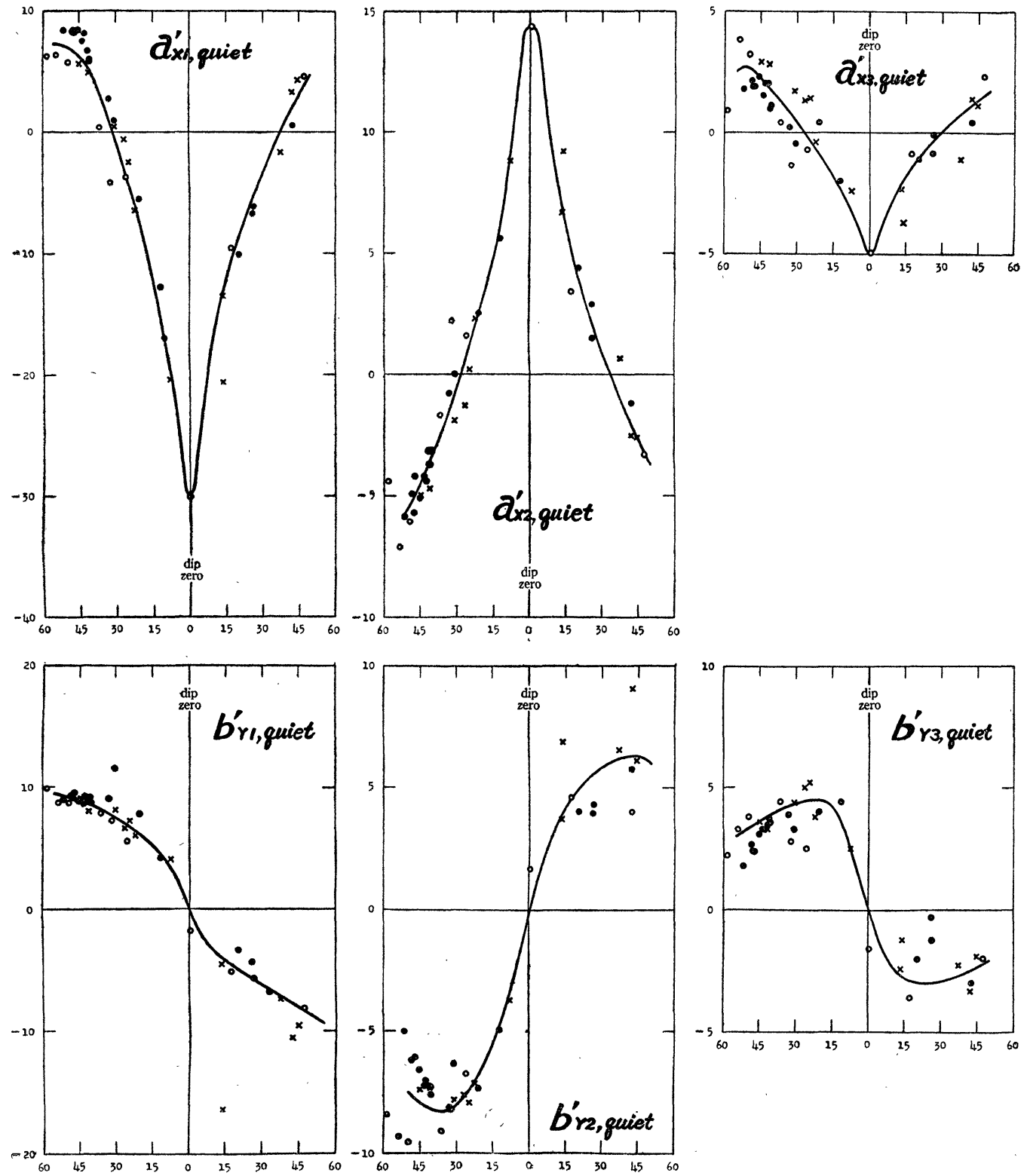

Fig. 8 Distributions of $a_{X}^{\prime}$ 's and $b_{Y}^{\prime}$ 's arranged on the modified latitude (which kegins from the point where dip is zero) 
a method, and we can see that the discrepancies between the different zones are almost ignored, compared with those of the previous paragraph (Figs. 6 \& 7), and the feature of the latitudinal distribution at the equatorial zone is made clear in spite of the lack of observation at this zone.

\section{Simple expression of the $S_{q}$-variation at the middle and the low latitudes}

On the analysis of the diurnal variation expressed by the harmonic coefficients, it is assumed that the geomagnetic force varies uniformly during a full 24 hours as shown in the following equations;

$$
\begin{aligned}
& \mathrm{X}=a_{X 0}+\sum_{m=1}^{p}\left(a_{X m} \cos m t+b_{X m} \sin m t\right) \\
& \mathrm{Y}=a_{Y 0}+\sum_{m=1}^{p}\left(a_{Y m} \cos m t+b_{Y m} \sin m t\right) \\
& \text { where } t \text { denotes local time (LT), } a \text { 's and } b \text { 's are functions of latitude, } \\
& \quad \text { and these relations are satisfied when } p=3 \text { as described in the } \\
& \quad \text { paragragh } 2 \text {. }
\end{aligned}
$$

But the apparent feature recorded on magnetograms shows that the main part of the variations on the quiet days occurs during the day-time only. In view of this opinion it may be thought that the Fourier coeffs. analysed over a full 24 hours are not an actual existence so far as the geomagnetic $S_{q}$-variation is concerned. As we see in Figs. $6 \& 7$, the feature of the latitudinal distribution of cosine-terms of X-comp. $\left(a_{X 1}^{\prime}, a_{X 2}^{\prime}\right.$ and $\left.a_{X 3}^{\prime}\right)$ have a certain similarity, i.e., these three show that the point of zero (where the potential is max. or min.) is situated at about $30^{\circ} \mathrm{N}$ and $S$ on $11^{\mathrm{h}}$ meridian in LT. As it is unnatural that three elements have the same phases about local time and reach maximum on the same latitude, the writer wishes to interpret this fact as one phenomenon. The writer proposes a scheme which shows sine or cosine during the day-time and zero during the night, as shown by the thick lines in Fig. 9. Indeed, at the middle and the low latitudes, the feature

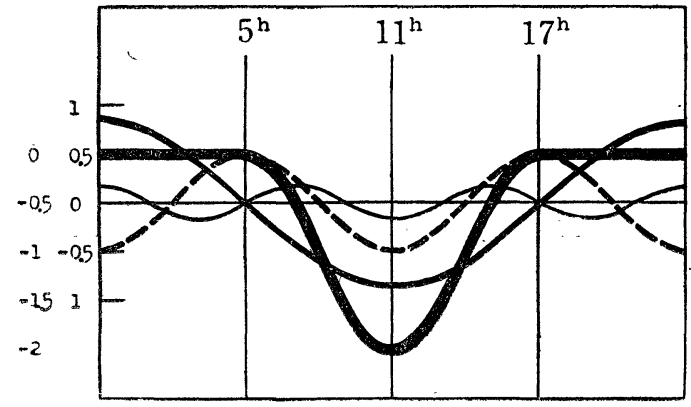

Fig. 9a Partial cosine-curve and its harmonics on a full 24 hours.

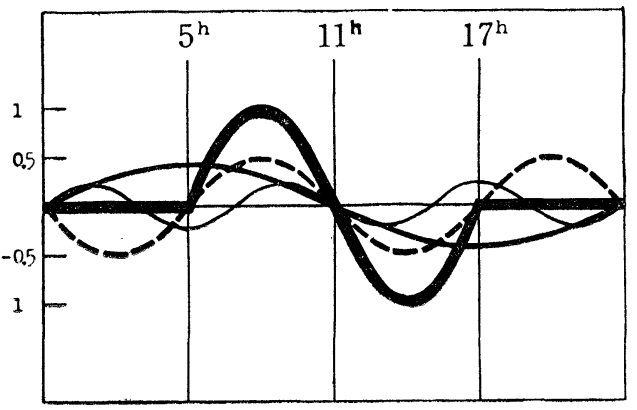

Fig. 9b Partial sine-curve and its harmonics on a full 24 hours.

of $\mathrm{X}$-variation on the quiet days is similar to the cosine-curve of this scheme (Fig. $9 \mathrm{a}$ ), and that of Y-variation sine-curve (Fig. 9b), and they are centred at $11^{\mathrm{h}}$ in LT., such as shown in the equation (3); 


$$
\mathrm{X}=\alpha_{0}{ }^{\prime}+\alpha_{1}{ }^{\prime} \cos \tau^{\prime} \quad \mathrm{Y}=\beta_{0}{ }^{\prime}+\beta_{1}{ }^{\prime} \sin \tau^{\prime}
$$

where $\tau^{\prime}$ is defined during $5^{\mathrm{h}}-17^{\mathrm{h}}$ and taken $\tau^{\prime}=0$ at $5^{\mathrm{h}}$, and $\tau^{\prime}=2 \pi$ at $17^{\mathrm{h}}$, then $\alpha_{0}{ }^{\prime}=-\alpha_{1}{ }^{\prime} \beta_{0}{ }^{\prime}=0$ by the assumption which $\mathrm{X}$ and $\mathrm{Y}$ are zero during the night $\left(17 \mathrm{~h}-5^{\mathrm{h}}\right)$.

$\sigma_{1}{ }^{\prime}$ and $\beta_{1}{ }^{\prime}$ are derived by the product of the conventional harmonic-coeffs. and the multiplying factor as follows;

$$
\begin{array}{ll}
a_{X 1}^{\prime}=0.85 \alpha_{1}{ }^{\prime} & b^{\prime}{ }_{Y 1}=0.44 \beta_{1}{ }^{\prime} \\
a_{X 2}^{\prime}=-0.5 \alpha_{1}{ }^{\prime} & b^{\prime}{ }_{Y 2}=-0.5 \beta_{1}{ }^{\prime} \\
a_{X 3}^{\prime}=0.17 a_{1}{ }^{\prime} & b_{Y 3}^{\prime}=0.24 \beta_{1}{ }^{\prime}
\end{array}
$$

where $a$ 's and $b$ 's ; coeffs of the conventional harmonic-analysis $\alpha_{1}^{\prime}$ and $\beta_{1}^{\prime}$; coeffs. (amplitudes) of the partial cosine- and sine-curves which are proposed here.

These relations are also shown in Fig. 9. Besides, the relations are confirmed by

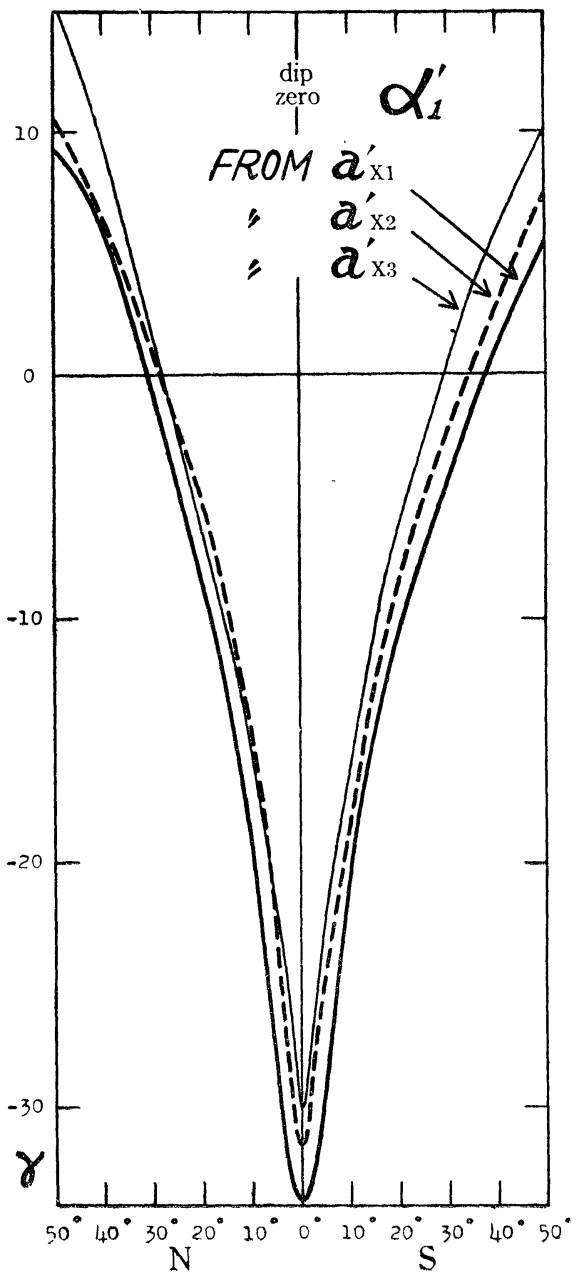

Fig. 10a Latitudinal distributions of $\alpha^{\prime}$, calculated by the eq. (4).

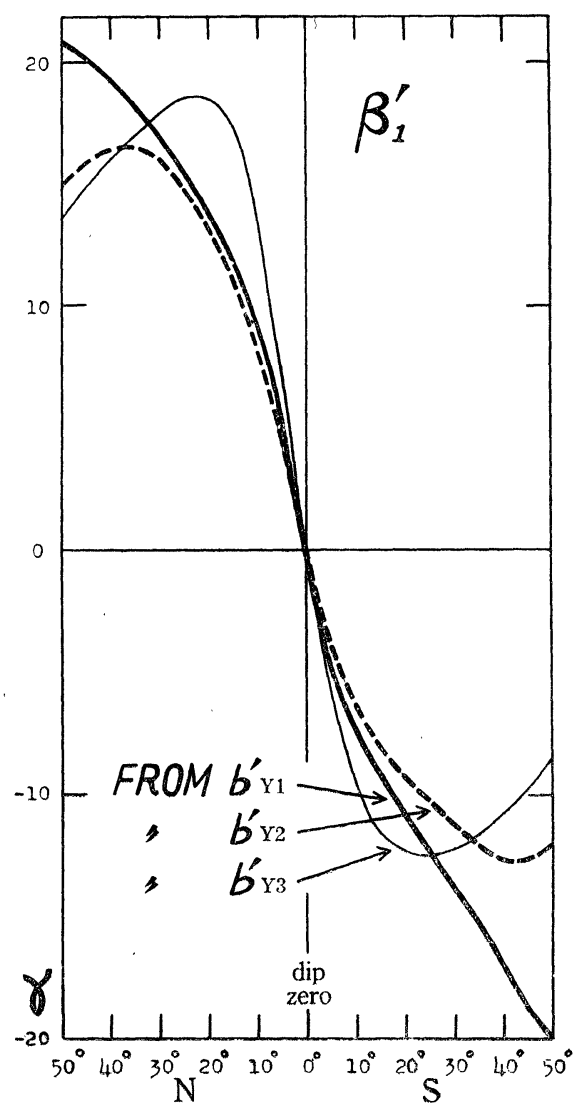

Fig. 10b Latitudinal distributions of $\beta^{\prime}$, calculated by the eq. (4) 
the fact that the three harmonics of the conventional one are predominant as mentioned in paragraph 2. But, in this case there are three relations for a sake of one coeff. $\boldsymbol{\sigma}_{1}{ }^{\prime}$ (or $\beta_{1}{ }^{\prime}$ ), then three independent values derived from the above relations have to coincide with each other. The latitudinal distributions of these three values thus obtained are shown in Fig. 10. Since by this figure the three diagrams which are obtained by the three relations are almost agreeable, then the question is solved as a whole. As a conclusion for these diagrams which are to coincide with each other, the writer adopts the weighted mean which is antiproportional to the multiplying factor of the equation (4), and it is shown in Fig. 11.

Strictly speaking, there is some disagreement between the three diagrams. This disagreement is caused by terms of the full 24 hours' waves as well as error in the assumption of the scheme. But as these values are of the same order of regional anomalies of the $S_{q}$-field, the writer wishes to adopt this scheme as the present course of analysis. Moreover this scheme is more convenient and reliable than the previous one for the reason given in the next paragraph.

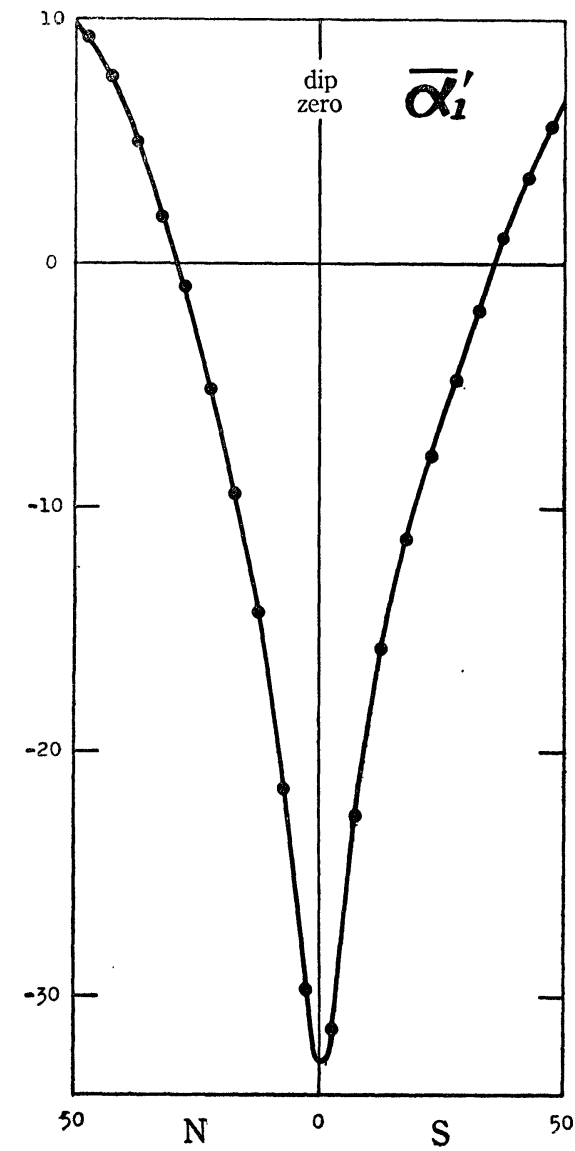

Fig. 11a Latitudinal distributions of $\alpha_{1}^{\prime}$. (weighted mean of three values in Fig. 10a)

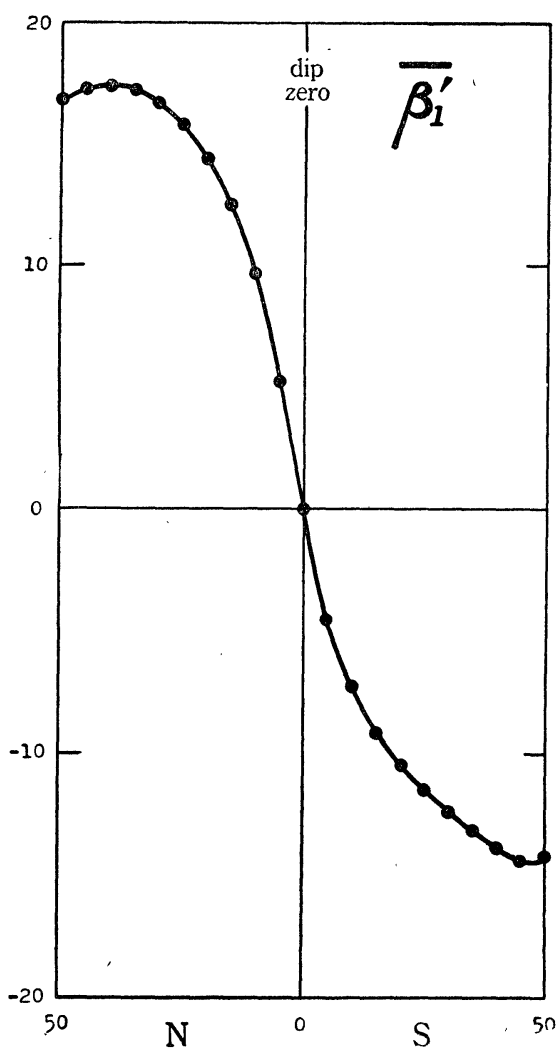

Fig. 11b Latitudinal distributions of $\beta_{1}^{\prime}$. (weighted mean of three values in Fig. 10b) 
The scheme proposed here brings up two questions; one concerns the discontinuities at $5^{\mathrm{h}}$ (near sunrise) and $17^{\mathrm{h}}$ (near sunset), and the other is the $\mathrm{X}$-values during the night. About these questions the writer wishes to give the following interpretation; this scheme is defined during the day-time only, where the physical state at $5^{\mathrm{h}}$ is equal to that at $17^{\mathrm{h}}$, and the phenomenon (supposed in this paper) is assumed to repeat itself as a period of 12 hours. Though the physical state during the night is not taken into consideration, $\mathrm{X}$ - and $\mathrm{Y}$-components are taken to be zero all through the night, and to have the same values as those at $5^{\mathrm{h}}$ and $17^{\mathrm{h}}$. Then, to answer the questions, it must be supposed that the zonal electric current which is proportional to $\sigma_{1}^{\prime}$ (which is a function of latitude) continues throughout the day. 6. Comparison between the coefficients derived from the $X$-component and from the Y-component

On the determination of the coeffs. of the potential function which expresses the geomagnetic diurnal-variation, Prof. Champman [6] adopts the values derived from the Y-comp. But Hasegawa's paper [2] expresses both values as derived from $\mathrm{X}$ and $\mathrm{Y}$, the final values of which are not yet published, but which can be taken in the writer's hand, as shown in Table $\mathrm{I}$ :

Table I

Main teams of the spherical harmonic representation of th $S_{q}$-field computed by $\mathrm{X}$ and $\mathrm{Y}$ components, after Hasegawa and Ota. (local-time-field, normalized, unit $10^{-5} \mathrm{c} . \mathrm{g.s}$.)

\begin{tabular}{cccccr}
\multicolumn{3}{c}{$V=R \sum_{m} \sum_{n}\left(A_{n}^{m} \cos m t+B_{n}^{m} \sin m t\right) P_{n}^{m}(\theta)$} & \multicolumn{2}{c}{$t$ : local time } \\
cceffs. & \multicolumn{2}{c}{$A_{n}^{m_{n}}$} & \multicolumn{2}{c}{$B_{n}^{m}$} \\
$m$ & $n$ & from X & from Y & from X & from Y \\
1 & 2 & 10.10 & 7.09 & -2.47 & -2.28 \\
3 & 3 & -4.96 & -4.89 & 1.61 & 2.02 \\
2 & 4 & 1.25 & 1.25 & -0.95 & -0.78
\end{tabular}

By this Table we can see the following results about their main terms: About the coeffs. of the first harmonic, those from $X$ are larger than those from $Y$. About those of the second harmonic, both values are agreeable with each other. About those of the third harmonic, those from $\mathrm{X}$ is a little larger than those from $\mathrm{Y}$. As to these disagreements, the writer is of the opinion that it is caused by the following reason; the multiplying factor for $a_{X 1}^{\prime}$ to secure $a_{1}^{\prime}$ is $1.18(1 / 0.85)$, and that for $b_{Y 1}^{\prime}$ to secure $\beta_{1}{ }^{\prime}$ is $2.23(1 / 0.44)$ as recognized by the equation (4). Then if the present scheme satisfy the conditions of the potential field by which the $S_{q}$-field is represented, coeffs. of the scheme derived from $X$ should take the same values as those derived from $\mathrm{Y}$. About the conventional analysis the coeffs. $A_{2}{ }^{1}$ and $B_{2}{ }^{1}$ from $\mathrm{X}$ are larger than those from $\mathrm{Y}$. Though the reduction-factor does not cancel this difference, as the writer has modified the latitude, this is almost removed. The multiplying factor of the second harmonic are $2(1 / 0.5)$ for $\mathrm{X}$ as well as $\mathrm{Y}$. This fact suggests that the second harmonic has a characteristic essentially the same as the scheme proposed here. As to the third harmonics, the writer would refrain from definite discussion, as their values are small compared with the first and the second. 
The potential by the present scheme is written as follows;

$$
\begin{aligned}
& V=R\left(A_{0}{ }^{\prime}+A_{1}{ }^{\prime} \cos \tau^{\prime}\right) \\
& A_{0}{ }^{\prime} \text { and } A_{1}{ }^{\prime} \text { are functions of latitude }
\end{aligned}
$$

then $\quad \mathrm{X}=\frac{\partial A_{0}{ }^{\prime}}{\partial \theta}+\frac{\partial A_{1}{ }^{\prime}}{\partial \theta} \cos \tau^{\prime}$

$$
\mathrm{Y}=(2 / \sin \theta)\left(A_{1}{ }^{\prime} \sin \tau^{\prime}\right) \text { as its definition }
$$

$A_{1}{ }^{\prime}$ and $\frac{\partial A_{1}{ }^{\prime}}{\partial \theta}$ are derived from $\mathrm{X}$ and $\mathrm{Y}$ independently, using the latitudinal distribution of $\alpha_{1}^{\prime}$ and $\beta_{1}^{\prime}$ (Fig. 11). In this calculation, values at every $5^{\circ}$ (in latitude) are measured by the diagram of Fig. 11. Fig. 12 shows the latitudinal distributions of $A_{1}{ }^{\prime}$ and $\frac{\partial A_{1}{ }^{\prime}}{\partial \theta}$ which have two diagrams respectively. By this figure it may be said that the features of the two diagrams derived from $\mathrm{X}$ and $\mathrm{Y}$ almost coincide

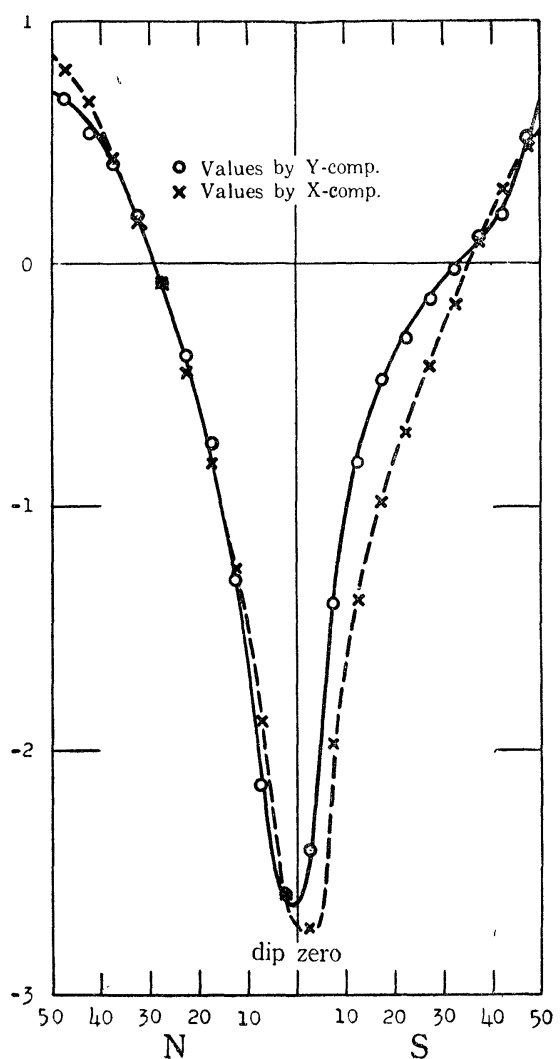

Fig. 12a Latitudinal distributions of $\partial A^{\prime}{ }_{1} / \partial \theta$, calculated by the eq. (3) and (5).

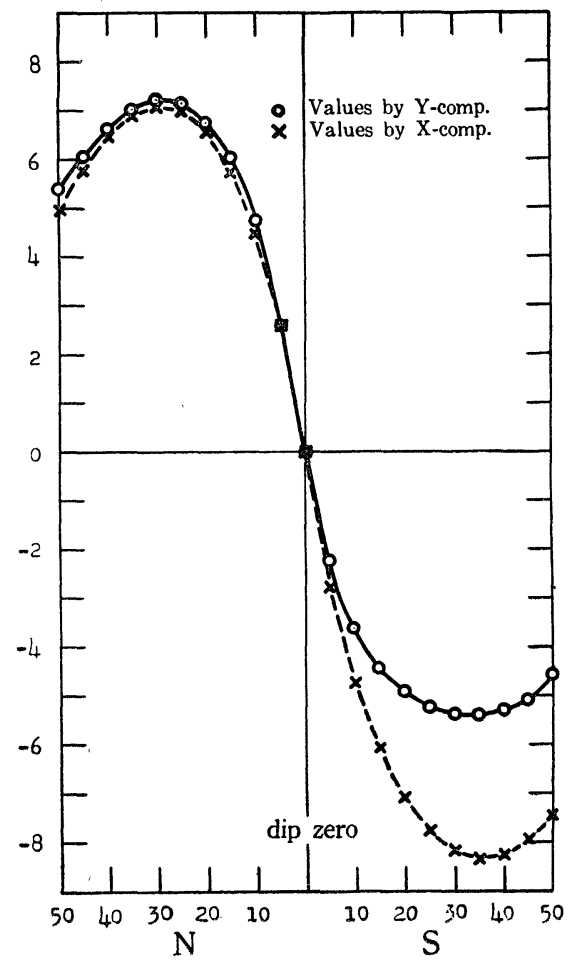

Fig. 12b Latitudinal distributions of $A^{\prime}{ }_{1}$, calculated by the eq. (3) and (5).

with each other in the northern hemisphere, but not in the southern hemisphere where observatories are sparse and regional anomalies of the $S_{q}$-field are easily affected in the investigation of world-wide phenomena. Therefore, the writer adopts the diagram of the northern hemisphere, in which mean of the values from $\mathrm{X}$ and $\mathrm{Y}$ (Fig. 12) is used, and assumes that the field is just asymmetrical in the both hemispheres. Fig. 13 shows the $S_{q}$-field thus obtained, and the amounts of the total 
current by this field is about 58000 ampere. This value is very agreeable with the previous results. It is also investigated that this field has not a non-potential part by the above discussions.

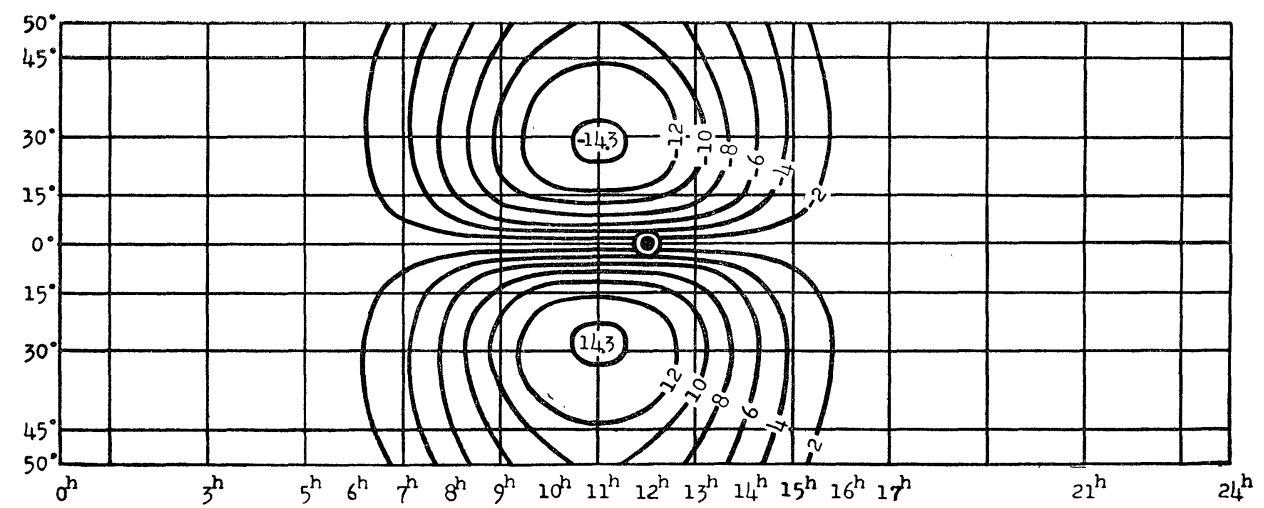

Fig. 13 Simplified $S_{q}$-field expressd by $V R\left(A^{\prime}{ }_{0}+A^{\prime}{ }_{1} \cos \tau^{\prime}\right)$ denotes the position of the sun. Unit : $6.4 \times 10^{3}$ c.g.s.

\section{Conclusion}

The present paper is a chapter of an analysis of the diurnal variation of the terrestrial magnetism and the preliminary settlement of the problem in which the regional anomalies, the axes of the variation and other elements related to securing the feature of the $S_{q}$-field at the middle and the low latitudes are discussed. The detailed discussions about those problems will be done in the next paper which will be published in near future.

The scheme of the $S_{q}$-variation proposed here is not a complete expression in mathematics, but is able to simplify it and will be service in the investigation of the conductivity and the motion of the upper atmosphere. It is clear that the $S_{q}$ field has no non-potential part, and it is worth examining whether the disturbing field have non-potential part or not.

The terms of $Z$-component are perhaps important in respect to this subject, but their regional-anomalies are so irregular that they could not be examined.

\section{Acknowledgment}

This work is one of the continuations of the statistical investigation commenced by Prof. M. Hasegawa since 1937, and the data used here are collected by him. The writer wishes to express his sincere thanks to Prof. Hasegawa for his guidance and to Dr. Y. Tamura for his encouragement in this work.

\section{References}

[1] T. Rikitake. I. Yokoyama and Y. Hishiyama; Bull. of the Earthq. Res. Inst., 30, 217 (1952); 31, 19 (1953).

[2] M. Hasegawa and M. Ota ; IATME Bull. No. 13, Trans. of Oslo Meeting, 426 (1950). 
[3] M. Ota; IATME Bull. No. 13, Trans. of Oslo Meeting, 438 (1950).

[4] M. Hirono; Jour. of Geomag. and Geoele., 2, 113 (1950).

[5] H. Maeda; in press.

[6] S. Chapman; Phil. Trans. A, 218, 23 (1919).

\section{Meeting of the Society of Terrestrial Magnetism and Electricity :}

The 14th General Meeting was held at the Kyoto University on Oct. 30Nov. 1, 1953.

Number of the Reports read at the Meeting:

Cosmic Rays, 9; Atmospheric Electricity, 9; Geomagnetism, 7 ; Rock Magnetism, 6; Radio Meteorology, 3; Ionosphere, 9.

Tanakadate Prize was awarded for the following excellent worker:

The 14th, Mr. K. Nagashima;

Theoretical Research on the Cosmic Ray Variation caused by the Electric Field.

The 15th General Meeting was held at the Tokyo University on May 2830, 1954.

Number of the Reports read at the Meeting:

Geomagnetism, 7; Ionosphere, 16; Cosmic Rays, 9; Rock Magnetism and Electricity, 7 ; Atmospheric Electricity, 14.

Tanakadate Prize was awarded for the following excellent worker:

The 15th, Mr. K. Hirao;

Radio Meteological Research in the Ultra Short Wave Propagation, especially on the Nocturnal Cooling Play. 